\title{
Study of Two-Dimensional Photonic Crystal Microcavities as a Function of Refractive Index
}

\author{
R. Moukhtari, A. Hocini* and D. Khedrouche \\ Laboratoire d'Analyse des Signaux et Systèmes, Department of Electronics, Mohamed Boudiaf University of M'sila, \\ B.P. 166, Route Ichebilia, M'sila, 28000 Algeria
}

\begin{abstract}
Quality factor of two-dimensional photonic crystals microcavity fabricated by $\mathrm{SiO}_{2} / \mathrm{ZrO}_{2}$ or $\mathrm{SiO}_{2} / \mathrm{TiO}_{2}$ in which the refractive index varied in the range of 1.51 to 1.58 has been investigated in the present paper. The structure studied consists of circular rods of radius $r$ embedded in air slab, while $a$ is the constant of triangular lattice. Finite difference time domain method with perfectly matched layers was used to calculate the transmission spectrum by FullWAVE software. The results of simulation clearly demonstrate the existences of resonance wavelength of which the position and the highest $Q\left(Q \approx 4 \times 10^{5}\right)$ value are strongly affected by refractive index. We demonstrate that the calculated $Q$ factor for the designed cavity increases by a factor of three relative for a cavity without increasing the refractive index.
\end{abstract}

DOI: 10.12693/APhysPolA.129.556

PACS/topics: 42.70.Qs, 04.30.Nk, 41.20.Jb, 42.82.-m

\section{Introduction}

Photonic crystals (PhCs) are artificial materials consisting of a periodic arrangement in which the dielectric constant (the refractive index) has a periodicity in one, two or three dimensions that provide control of light at a wavelength scale [1]. Photonic crystal devices with one-dimensional (1D), two-dimensional (2D) and threedimensional (3D) structures have been fabricated by wet dry etching, wafer bonding, self-assembled method and electron-beam lithography [2]. Since the introduction of the concept of photonic crystals by Yablonovich [3] and John [4], they have attracted much interest due to their potential for controlling the propagation of light for its photonic band gap (PBG) and/or light diffraction properties [5]. In recent years, 2D (PhCs) composed of air holes drilled in a dielectric slab and structured in a triangular lattice are most commonly used due to fabrication difficulty of other types of photonic crystals (3D). In the other hand, 2D photonic crystals have a great ability for integration [6]. Consequently, they have been widely studied and analyzed as the building blocks to realize functional devices for optical networking, waveguides, photonic crystal fibers, photonic cavities, and optoelectronics applications.

Photonic crystal microcavities have been studied widely for their ability to confine light selectively at different operating wavelengths. This property is clearly highly desirable in the application areas of wavelength division multiplexing and filtering. Photonic crystal microcavities are typically characterized by two key quantities, the quality factor, a measure of the photon lifetime for

\footnotetext{
* corresponding author; e-mail: abdesselam.hocini@univ-msila.dz
}

the relevant optical cavity mode, and the modal volume, a measure of the spatial extent and energy density of the mode. Several authors have reported photonic crystal cavities with high $Q$-values and various techniques have been used to couple light into the cavities [7]. The realization of a high- $Q$ microcavity at optical wavelength has many important technological consequences. According to the Purcell effect, the rate of spontaneous emission can be greatly altered inside a high- $Q$ cavity [8]. A high- $Q$ cavity also operates as a band pass filter for on-off optical switching [9]. Its ability to confine light strongly will lead to the creation of high- $Q$ cavities at optical $\lambda$ and with a small size. Moreover, its sub- $\lambda$ property permits great geometrical flexibility in designing microcavities. By using different types and combinations of local defects, one can vary both a cavity's resonant frequency and its mode symmetry. Whereas a three-dimensional (3D) photonic crystal is ideal for creating high- $Q$ cavities [10], one and two-dimensional (1D and 2D) photonic crystals have the advantage of simplicity of fabrication [11].

We propose a PC microcavities device that consists of a photonic crystal air slab, with a periodic triangular lattice of rods in $\mathrm{SiO}_{2} / \mathrm{ZrO}_{2}$ or $\mathrm{SiO}_{2} / \mathrm{TiO}_{2}$ characterized with low refractive index which varied in the range of 1.51 to 1.58 at $\lambda=1.55 \mu \mathrm{m}$ [12], using the finite difference time domain (FDTD) method assisted by group theory in the time domain [13]. The $\mathrm{PhC}$ microcavities $\left(H_{1}\right)$ are obtained by removing a single rod of the $2 \mathrm{D}$ photonic crystal structure. We simulate this cavity that $\mathrm{PhC}$ structure ensures high transmission efficiency and we report a theoretical study of the quality factor and the parameters that affect this later. In the first part of this work, we have demonstrated the existence of resonance wavelength in 2D photonic crystals cavity fabricated with $\mathrm{SiO}_{2} / \mathrm{ZrO}_{2}$ or $\mathrm{SiO}_{2} / \mathrm{TiO}_{2}$ [14]. In the second part, we have investigated the effects of the contrast on the $Q$ factor. 


\section{Photonic crystal structure}

Photonic crystals, which prohibit the propagation of light for frequencies within a band gap, have enabled exciting new ways of controlling light and of constructing integrated optical devices [15]. The photonic crystal considered here is a $2 \mathrm{D}$ triangular lattice structure of air slab, as shown in Fig. 1a, however, the triangular lattice is very suitable to studying photonic band gap properties. The triangular lattice allows the opening of $2 \mathrm{D}$ photonic band gap, presents a good compromise namely for high filling factors and its low sensitivity to the incidence angle compared to the square lattice [12].

Figure 1a shows the top view of a two-dimensional photonic crystals $(2 \mathrm{D} \mathrm{PhC}$ ) with rods in air triangular lattice. The rod represented by the solid cylinders corresponding to $\left(\mathrm{SiO}_{2} / \mathrm{TiO}_{2}\right.$ or $\left.\mathrm{SiO}_{2} / \mathrm{ZrO}_{2}\right)$ material of low refractive index which varied in the range of 1.51 to 1.58 at $\lambda=1.55 \mu \mathrm{m}$. We can produce rods of the refractive index which is within the range from the refractive index of pure silica $(N=1.4)$ to the refractive index of titania $(N=2.3)[16,17]$. The rods of low refractive indexes afford the possibility to produce planar systems, which are compatible with optical telecommunication. The radius of each rod are chosen to be $0.23 \mu \mathrm{m}$, where $a$ is the lattice constant of the $2 \mathrm{D}$ structures and it equals $0.75 \mu \mathrm{m}$. The two parameters $r$ and $a$ are chosen for yield of a large photonic band gaps.
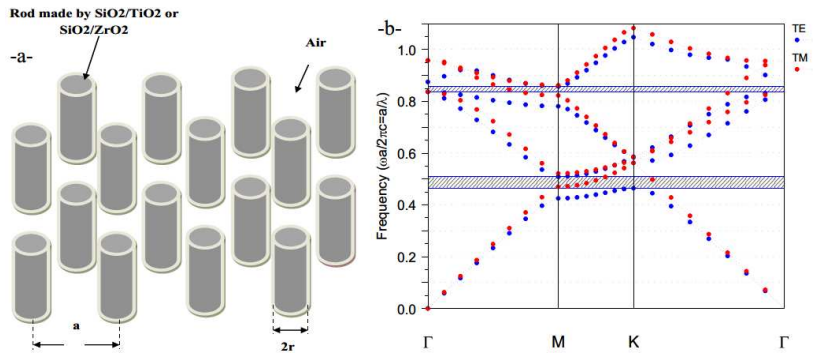

Fig. 1. (a) Disconnected structure of 2D photonic crystal made with $\mathrm{SiO}_{2} / \mathrm{TiO}_{2}$ or $\mathrm{SiO}_{2} / \mathrm{ZrO}_{2}$ rods in air, (b) dispersions curves and band-gaps for TM and TE polarizations for the 2D lattice without defects.

Dispersion diagram showing normalized frequency versus the wave vector for transverse electric (TE) and transverse magnetic (TM) modes of the $2 \mathrm{D}$ photonic crystal is given in Fig. 1b. It has been calculated along the $\Gamma-X-M-\Gamma$ edge for the Brillouin zone by employing a $2 \mathrm{D}$ plane wave expansion (PWE) method. The band diagrams show a two frequency band gap for the TE polarized modes but no gap for TM modes with this refractive index contrast and relatively small $r / a$. In the TE band diagram the fundamental band gap is centered near a wavelength of 1.537. It extends between to wavelength range $1.469-1.605 \mu \mathrm{m}$ for the waves with TE polarization for the refractive index $n=1.58$.

\section{Numerical analysis and discussion}

This section presents the influence of refractive index contrast, which was changed in order to predict the highest quality factor $Q$ for improve the transmission efficiency. The numerical tools used for our simulations are based on the two-dimensional finite difference time-domain method (FDTD) (RSoft Design Group, FullWAVE) [18].

The structure of our 2D PC cavity is shown in Fig. 2, with one missing rod in the center, where the number of circular rods considered for both $X$ and $Z$ directions are 25. This cavity allows for very high quality factors while maintaining a size of the order of optical wavelength.

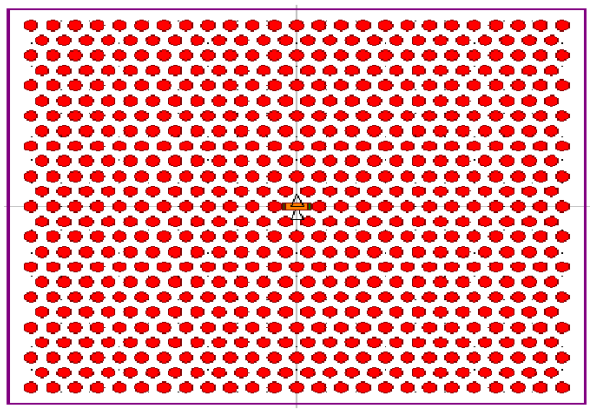

Fig. 2. Sight of top of the $H_{1}$ cavity made by removed one rod.

We begin our consideration by calculation the transmission spectra for a variety of refractive index $(n)$ in the range 1.51 to 1.58 . Figure 3 shows the spectral response of the microcavity formed by removing one rod in air obtained with the FDTD method of the impulse response for different values of refractive index $n=1.51$ to 1.58. The spectrum calculated by TE polarization can be observed as one cavity mode for different values of refractive index. We will call the mode of mode resonance wavelength.
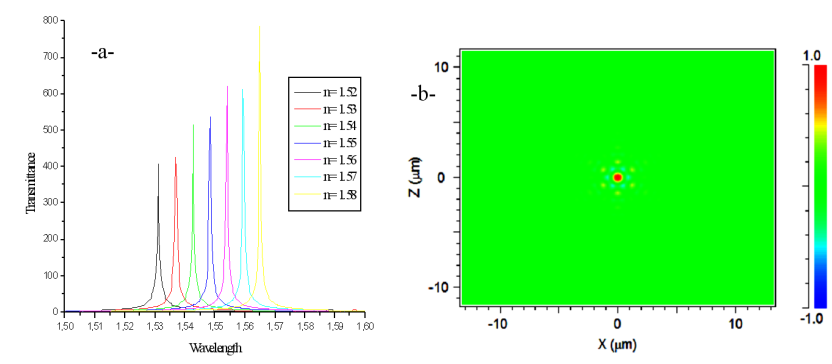

Fig. 3. (a) Frequency response of the $H_{1}$ microcavity formed by removing one rod triangular lattice as a function of refractive index, (b) the intensity field distribution for the microcavity at resonant wavelength $1.565 \mu \mathrm{m}(n=1.58)$.

In order to show the location of resonance wavelength and quality factor, full wave software is used to simulate the transmission spectrum for different values of refractive index $n=1.51$ to 1.58 . Figure 3 reports the simulations. 
The computational method used is based on a 2D FDTD method algorithm. Perfectly matched layers (PML) conditions have been considered in the calculations to ensure no back reflection in the limit of the analyzed region. This crystal is lighted by a Gaussian wave under normal incidence with a transverse electric (TE) polarized. The time step is chosen to 0.01. Note that it might be necessary to reduce the time step below the stability limit when simulating metals since the courant condition can change in this case.

The results show that more refractive index increase the resonance wavelength shifts towards the right $\lambda_{0}=$ 1.525 to $1.565 \mu \mathrm{m}$, and quality factor $Q=\left(\lambda_{0} / \Delta \lambda\right)$ varied from $Q=1.3 \times 10^{5}$ and $V$ of $1.38\left(\lambda_{0} / n\right)^{3}$ to $4 \times 10^{5}$, $V$ of $1.05\left(\lambda_{0} / n\right)^{3}$.

Figure 4 presents the variations of resonance wavelength and quality factor as a function of refractive index.
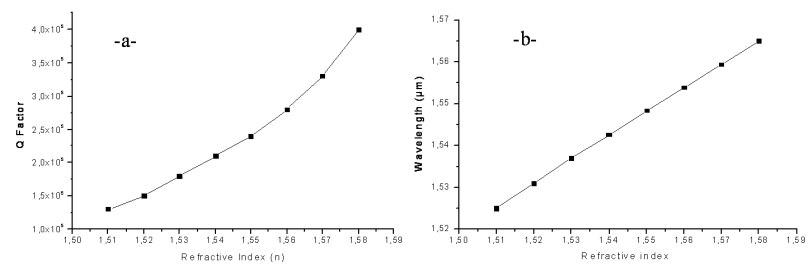

Fig. 4. (a) Frequency response of the $H_{1}$ microcavity formed by removing one rod triangular lattice as a function of refractive index, (b) the intensity field distribution for the microcavity at resonant wavelength $1.565 \mu \mathrm{m}(n=1.58)$.

This quality factor reflects the ability of the cavity to trap light and represents a measure of loss. At resonance, the photon undergoes multiple reflections between the two mirrors defining the cavity and comes out of it after a while that can be considered as the life of the photon in the cavity.

We begin our consideration by calculation the transmission spectra by increasing the refractive index. We show the dependence of the quality factor and the resonance wavelength as a function of the symmetric than the refractive index increases. After careful scanning of refractive index $n$, we find the highest $Q$ value $Q=4 \times 10^{5}$, the $Q / V$ of the obtained nanocavity is as large as $3.6 \times 10^{5}\left(\lambda_{0} / n\right)^{-3}$ with resonant frequency $1.565 \mu \mathrm{m}$ which can be achieved at $n=1.58$.

\section{Conclusion}

In conclusion, a photonic microcavity of twodimensional photonic crystals formed by rods made with $\mathrm{SiO}_{2} / \mathrm{ZrO}_{2}$ or $\mathrm{SiO}_{2} / \mathrm{TiO}_{2}$ embedded in air slab and characterized by low refractive index which change in the range of 1.51 to 1.58 have been investigated and reported in the present paper. The different results of simulation obtained by FDTD method in Fullwave software clearly demonstrated the existence of the resonance wavelength of triangular lattice structure. The results show that the refractive index has a great effect on the position of resonance wavelength and highest $Q$ value. Thus we reveal that a maximum absolute quality factor for this structure is achieved for $n=1.58$.

In future works, the magneto photonic cavity of such structures will be considered.

\section{References}

[1] J.D. Joannopoulos, R.D. Meade, J.N. Winn, Photonic Crystals, Princeton Univ. Press, Princeton 1995.

[2] A. Benmerkhi, M. Bouchemat, T. Bouchemat, N. Paraire, J. Mater. Sci. Eng. 15, 012094 (2012).

[3] E. Yablonovitch, J. Phys. Rev. Lett. 58, 2059 (1987.

[4] S. John, Phys. Rev. Lett. 58, 2486 (1987).

[5] A. Harhouz, A. Hocini, J. Electromagn. Wave Appl. 29, 659 (2015).

[6] J.M. Lourtioz, H. Benisty, V. Berger, J.M. Gerard, D. Maystre, A. Tchelnokov, Photonic Crystals, Springer, Berlin 2005.

[7] S.Y. Lin, E. Chow, S.G. Johnson, J.D. Joanopolous, J. Opt. Lett. 26, 1903 (2001).

[8] E. Yablonovitch, J. Opt. Soc. Am. B 10, 283 (1993).

[9] P.R. Villeneuve, D.S. Abrams, S. Fan, J.D. Joannopoulos, J. Opt. Lett. 21, 2017 (1996).

[10] S.Y. Lin, J.G. Fleming, M.M. Sigalas, R. Biswas, K.M. Ho, J. Phys. Rev. 59, R15579 (1999).

[11] D.R. Smith, R. Dalichaouch, N. Kroll, S. Schultz, S.L. McCall, P.M. Platzman, J. Opt. Soc. Am.10, 314 (1993).

[12] A. Kahlouche, A. Hocini, D. Khedrouche, J. Comput. Electron. 6, 0559 (2014).

[13] M. Okano, S. Noda, Phys. Rev. B 70, 125105 (2004).

[14] C.J. Brinker, G.W. Scherer, Sol-Gel Science, Academic Press, Boston 1990.

[15] O. Bouleghlimat, A. Hocini, Phys. Scr. 89, 105502 (2014).

[16] A. Hocini, M. Bouras, H. Amata, J. Opt. Mater. 35, 1669 (2013).

[17] A. Hocini, A. Bouchelaghem, D. Saigaa, M. Bouras, T. Boumaza, M. Bouchemat, J. Comput. Electron. 12, 50 (2013).

[18] RSoftDesign Group, FullWAVE v3.0.1., Inc. 200 Executive Blvd., Ossining, NY 10562. 